\title{
Negative-resistance models for parametrically flux-pumped superconducting quantum interference devices
}

\author{
Kyle M Sundqvist ${ }^{1 *}$ and Per Delsing ${ }^{2}$
}

\author{
*Correspondence: \\ kyle.sundqvist@gmail.com \\ 'Electrical and Computer \\ Engineering, Texas A\&M University, \\ College Station, TX 77843, USA \\ Full list of author information is \\ available at the end of the article
}

\begin{abstract}
A Superconducting QUantum Interference Device (SQUID) modulated by a fast oscillating magnetic flux can be used as a parametric amplifier, providing gain with very little added noise. Here, we develop linearized models to describe the parametrically flux-pumped SQUID in terms of an impedance. An unpumped SQUID acts as an inductance, the Josephson inductance, whereas a flux-pumped SQUID develops an additional, parallel element which we have coined the "pumpistor." Parametric gain can be understood as a result of a negative resistance of the pumpistor. In the degenerate case, the gain is sensitive to the relative phase between the pump and signal. In the nondegenerate case, gain is independent of this phase.

We develop our models first for degenerate parametric pumping in the three-wave and four-wave cases, where the pump frequency is either twice or equal to the signal frequency, respectively. We then derive expressions for the nondegenerate case where the pump frequency is not a multiple of the signal frequency, for which it becomes necessary to consider idler tones that occur. For the nondegenerate three-wave case, we present an intuitive picture for a parametric amplifier containing a flux-pumped SQUID where current at the signal frequency depends upon the load impedance at an idler frequency. This understanding provides insight and readily testable predictions of circuits containing flux-pumped SQUIDs.
\end{abstract}

Keywords: parametric amplifiers; SQUIDs; Josephson devices

\section{Introduction}

Parametric amplifiers are attractive in that they can in principle amplify a signal while only adding a minimum of noise [1]. From this point of view, parametric amplifiers may be divided into two groups; phase sensitive amplifiers which amplify only one of the incoming quadratures, and phase insensitive amplifiers which amplify both quadratures, thereby preserving the phase of the signal. A phase sensitive amplifier can in principle amplify the signal without adding any noise. The minimum noise added by a phase insensitive amplifier corresponds to half a quantum of the amplified frequency, $\hbar \omega / 2$.

In a parametric amplifier, some parameter of the system must be varied in time. By pumping the system, i.e. modulating that parameter at one frequency, it is possible to amplify a signal at a different frequency. Power is transferred from the pump frequency to the signal frequency.

D 2014 Sundqvist and Delsing; licensee Springer on behalf of EPJ. This is an Open Access article distributed under the terms of the Creative Commons Attribution License (http://creativecommons.org/licenses/by/2.0), which permits unrestricted use, distribution, and reproduction in any medium, provided the original work is properly cited. 
Parametric amplifiers can be realized in a large number of systems, both in optics and in electronics. A typical example in optics is a fiber-based amplifier where the refractive index of the fiber material is modulated by the pump. In other systems utilizing varactor diodes, it is the nonlinear diode capacitance which is modulated. Varactor diodes are typically used at frequencies ranging from radio to $\mathrm{THz}$ frequencies.

Superconducting circuits can also be used to build parametric amplifiers in the microwave domain. The use of parametric amplifiers with Josephson junctions was pioneered by several researchers in the 1970s [2-6], as well as Bernard Yurke in the 1980s $[7,8]$. Josephson junctions are used as parametric inductances, and may be pumped either by a time varying current through the junction [9-11], or in a SQUID geometry by a time-varying magnetic flux [11-14]. Alternatively the kinetic inductance of a thin superconductor can be used as the parametric component $[15,16]$.

Parametric amplifiers based on superconducting devices have recently regained interest because of the need for better amplifiers for qubit readout and microwave quantum optics. The utility of these amplifiers have been demonstrated in a number of experiments showing single shot qubit readout [17], quantum feedback [18], vacuum squeezing [19], and in determining the statistics of nonclassical photon states [20]. There are two major advantages of superconducting parametric amplifiers: (i) they have very low dissipation, and (ii) they have well characterized and engineer-able properties. This makes it possible to design well functioning parametric amplifiers with good gain and little added noise $[9,21]$.

To understand and implement a parametric amplifier, one often needs to solve a system of coupled equations where it may be difficult to fully appreciate the amplifier's overall properties. Along with the resurgent use of parametric amplifiers as applied to quantum systems, a quantum optics formalism is also typically adopted to explain the amplifier.

By contrast, we recently presented [14] a linearized impedance model for a flux-pumped SQUID following the engineering formalism [22-24] developed for (classical) varactor diodes in the 1960s and 1970s. While a similar formalism had also been utilized for early treatments of Josephson junction parametric amplifiers [4], this had not been applied to the flux-pumped SQUID. The flux-pumped SQUID can be represented as a parallel combination of a Josephson inductance and an additional circuit element which we named the "pumpistor." The pumpistor has the frequency dependence of an inductance, but it is an inductance which is complex. The phase of this complex inductance (or impedance) depends on the phase angle of the pump relative to the signal. By properly adjusting the pump, the pumpistor can act as a negative resistance. Thus, it can provide gain in the circuit. In this recent paper, we treated only the three-wave degenerate case, i.e. where the pump is applied at exactly twice the signal frequency.

In this work, we extend this pumpistor model. We revisit the three-wave degenerate case to include higher-order saturation effects. We also explore the four-wave degenerate case, which couples to the pump at higher order. Perhaps most importantly, we also treat the nondegenerate case, where the pump frequency is not a multiple of the signal frequency. Here a matrix formalism provides for the exploration of many types of nondegenerate frequency mixing, which, in addition to gain as a negative resistance, also describes upand down-conversion of a signal. 


\section{The current response of a simple dc SQUID}

In this section, we briefly review the relations between external magnetic flux, effective junction phase, and series current in a dc SQUID. In this work, we refer to a dc SQUID simply as a "SQUID," and we consider it free of parasitic internal impedances. To begin, we first consider a single Josephson junction in order to introduce the Josephson relations due to the $d c$ and $a c$ Josephson effects [25].

\subsection{Current and voltage in a simple Josephson junction}

In a Josephson junction, the $d c$ Josephson effect denotes the relation between the phase difference $\phi_{J}$, i.e., the difference in phase between the superconducting order parameters on either side of the junction, and the current $I$ which flows through the junction. This is given by

$$
I=I_{c} \sin (\phi)
$$

Here, $I_{c}$ is the critical current for this single Josephson junction, which is its maximum allowed super-current. The ac Josephson effect relates the time derivative of the phase difference to the voltage, $V$, across the junction.

$$
V=\left(\frac{\Phi_{0}}{2 \pi}\right) \frac{d \phi}{d t}
$$

where, $\Phi_{0} \equiv h /(2 e)$ is the superconducting flux quantum. By taking the time derivative of Eq. (1) and combining it with Eq. (2), we see that the Josephson junction acts like an inductor, $d I / d t=V / L_{J}$, with the Josephson inductance

$$
L_{J}=\frac{\Phi_{0}}{2 \pi I_{c} \cos \phi}
$$

\subsection{Extending the Josephson relations to a SQUID}

Placing two Josephson junctions ("1" and "2") in parallel, we form a SQUID, where the currents combine as a sum. We adopt the sign conventions suggested in Ref. [26].

$$
I=I_{c 1} \sin \left(\phi_{1}\right)-I_{c 2} \sin \left(\phi_{2}\right)
$$

Going around the loop and returning to the same point, the phase can only subtend multiples of $2 \pi$. We therefore find a quantization condition for the superconducting loop flux. We regard the phase differences to occur only at the two Josephson junctions, i.e., neglecting the inductance of the loop. Furthermore we assume that the two junctions are equal, $I_{c 1}=I_{c 2}=I_{c} / 2$, and we define the SQUID phase to be $\phi=\left(\phi_{1}-\phi_{2}\right) / 2$. Then we arrive at the SQUID current,

$$
I=I_{c} \cos \left(\pi \frac{\Phi_{\text {ext }}}{\Phi_{0}}\right) \sin (\phi)
$$

We see that the SQUID acts just like a Josephson junction, but with a critical current tunable by the external magnetic flux $\Phi_{\text {ext }}$. Note that our choice of sign convention following Ref. [26] eliminates the need for taking the absolute value of the quantity $\cos \left(\pi \Phi_{\text {ext }} / \Phi_{0}\right)$ 
in Eq. (5). This is not the case in the definition commonly used in other very good and popular references $($ e.g., $[27,28])$. In any case, for this work we consider only the situation where $\left|\Phi_{\text {ext }} / \Phi_{0}\right|<|1 / 2|$. Here, the quantity corresponding to $\cos \left(\pi \Phi_{\text {ext }} / \Phi_{0}\right)$ is always positive regardless of convention.

Thus, we recover a device phenomenology similar to the single Josephson junction depicted in Eqs. (1) and (2). Specifically, the SQUID acts as a tunable inductance such that

$$
L_{J}=\frac{\Phi_{0}}{2 \pi I_{c} \cos \left(\pi \frac{\Phi_{\text {ext }}}{\Phi_{0}}\right) \cos \phi}
$$

In this section, we have defined the system of a SQUID by current and voltage relations similar to a single Josephson junction. We found the SQUID to be tunable by an externally applied magnetic flux. Using this framework, in the next section we examine the SQUID circuit response to a magnetic flux, applied dynamically.

\section{The signal impedance of a SQUID, subject to a dynamically pumped external magnetic flux}

We investigate how a SQUID responds as an impedance due to the presence of a periodic perturbation of the external magnetic flux. To this end, we assume the external flux is of the following form.

$$
\Phi_{\mathrm{ext}}=\Phi_{\mathrm{dc}}+\delta \Phi
$$

Here $\Phi_{\mathrm{dc}}$ is a static ("quiescent") magnetic flux, and we use a time-dependent perturbation of the form $\delta \Phi=\Phi_{\mathrm{ac}} \cos \left(\omega_{3} t+\theta_{3}\right)$.

For convenience of notation, we define these following normalized flux amplitudes.

$$
\begin{aligned}
& F=\pi \frac{\Phi_{\mathrm{dc}}}{\Phi_{0}} \\
& \delta f=\pi \frac{\Phi_{\mathrm{ac}}}{\Phi_{0}}
\end{aligned}
$$

\subsection{An aside regarding labels and conventions}

For clarity, we take the opportunity to introduce a handful of electromagnetic disturbances necessary to understand our system. These small-signal disturbances occur at different frequencies. We follow the nomenclature for frequency terms as presented by Blackwell and Kotzebue [22].

Regarding frequencies and how we label them, in this work we consider at most six frequencies due to possible mixing effects. Foremost, we consider a "signal" which exists at a frequency assigned to index 1. For a parametric amplifier, the signal frequency serves as the frequency of both the input and output of the device. In this case, we determine both the small-signal current and voltage components at this same signal frequency. This gives us a "signal impedance" upon which we base our subsequent reasoning. Some driven "pump" disturbance occurs at a frequency of index 3. This pump frequency corresponds to the frequency at which the SQUID is driven externally. The pumping of the SQUID provides for a nonlinear interaction to occur. Another frequency we consider is the "idler" frequency. An idler response comes about due to the nonlinear mixing between signal 
Table 1 Our convention for the frequencies involved in mixing effects

\begin{tabular}{lll}
\hline (Angular) frequency & Designation & Relation \\
\hline$\omega_{1}$ & "signal" & $\omega_{1}$ \\
$\omega_{2}$ & "idler" (three-wave difference) & $\omega_{3}-\omega_{1}$ \\
$\omega_{3}$ & "pump" & $\omega_{3}$ \\
$\omega_{4}$ & "idler" (three-wave sum) & $\omega_{3}+\omega_{1}$ \\
$\omega_{5}$ & "idler" (four-wave difference) & $2 \omega_{3}-\omega_{1}$ \\
$\omega_{6}$ & "idler" (four-wave sum) & $2 \omega_{3}+\omega_{1}$ \\
\hline
\end{tabular}

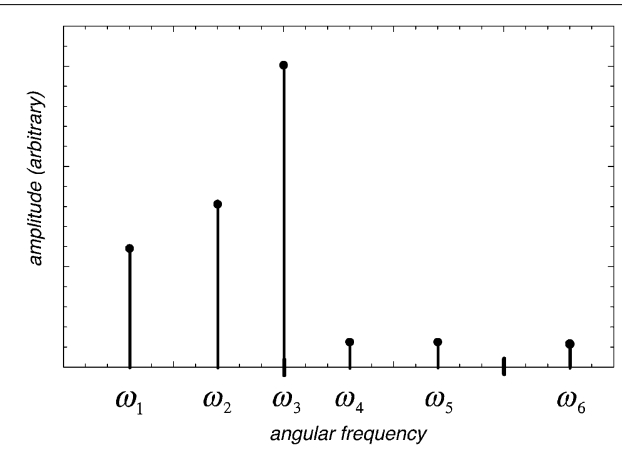

Figure 1 This figure depicts the mixing terms we consider pertinent. The signal frequency is at (angular) frequency $\omega_{1}$, and the pump frequency is $\omega_{3}$. The amplitudes are arbitrary.

and pump. In the general case, we need to provide for the possibility for the idler response to exist, even if it remains as an internal state variable (serving neither as an externally accessible input or output to the circuit). Among the various topologies which allow frequency mixing, an idler tone occurs at a frequency that is some linear combination of the signal and pump frequencies. In this work we delineate an idler as either a sum or a difference between signal and pump frequencies, for either the three-wave or four-wave case. An underlying principle of the parametric amplifier is that (some portion of) the power absorbed at the pump frequency is transferred to signal and idler frequencies, allowing for an amplified response.

We list all considered mixing frequencies in Table 1, and provide a depiction in Figure 1.

\subsection{Small-signal disturbances and the modulated SQUID current}

We consider different types of electromagnetic disturbances in the SQUID. Generally, we may account for voltage, current, junction phase, and magnetic flux. We have accounted for magnetic flux by Eq. (7). Consider also a general, small-signal response of the voltage, current, and junction phase at any of the six frequencies of Table 1. We assume ideal, sinusoidal tones.

$$
\begin{aligned}
v_{n}(t) & =\frac{1}{2} V_{n} e^{j \omega_{n} t}+\frac{1}{2} V_{n}^{*} e^{-j \omega_{n} t} \quad n \in\{1,2, \ldots, 6\} \\
i_{n}(t) & =\frac{1}{2} I_{n} e^{j \omega_{n} t}+\frac{1}{2} I_{n}^{*} e^{-j \omega_{n} t} \quad n \in\{1,2, \ldots, 6\} \\
\phi_{n}(t) & =\frac{1}{2} \tilde{\phi}_{n} e^{j \omega_{n} t}+\frac{1}{2} \tilde{\phi}_{n}^{*} e^{-j \omega_{n} t} \quad n \in\{1,2, \ldots, 6\}
\end{aligned}
$$

The amplitudes $V_{n}, I_{n}$, and $\tilde{\phi}_{n}$ are complex. Eqs. (10)-(12) also demonstrate that we have adopted the electrical engineering convention for complex number, $j$, rather than the 
physics convention, $i$, leading to a sign convention opposite of what one would find in the quantum optics literature.

The SQUID current is directly related to the junction phase by the dc Josephson effect as in Eq. (5). Note that we specify the current as two multiplicative terms; the "flux" term, and the "phase" term.

$$
i(t)=\underbrace{I_{c} \cos \left(\pi \Phi_{\text {ext }}(t) / \Phi_{0}\right)}_{\text {"flux" term }} \underbrace{\sin (\phi(t))}_{\text {"phase" term }}
$$

When treating these dynamics involving sinusoids, a common approximation is to implement Fourier-Bessel expansions [27]. However, a simple Taylor expansion recovers the same result as a Fourier-Bessel expansion when approximating Bessel functions in their small-signal limit.

We take separate series expansions of the two multiplied terms of Eq. (13). First, we expand the "flux" term. We use the flux-perturbation variable $(\delta \Phi)$ of Eq. (7), which was specified to be driven at the pump frequency $\left(\omega_{3}\right)$. To first order, we find the following.

$$
I_{c} \cos \left(\pi \Phi_{\text {ext }}(t) / \Phi_{0}\right) \approx I_{c}\left[\cos (F)-\sin (F) \delta f \cos \left(\omega_{3} t+\theta_{3}\right)\right]
$$

In some cases, such as when we consider saturation effects due to large flux amplitudes, we will expand this term to higher order.

Next, we expand the "phase" term of Eq. (13). We use simply $\sin [\phi(t)] \approx \phi(t)$, although we also include the cubic term in cases where we consider saturation effects due to junction phase. In the linear limit we consider the "phase" term to be the superposition of contributions from the six considered frequencies, $\phi(t)=\sum_{n=1}^{6} \phi_{n}(t)$, with $\phi_{n}(t)$ taken from Eq. (12).

The total SQUID current can now be approximated as the following.

$$
i(t) \approx \underbrace{\left\{I_{c}\left[\cos (F)-\sin (F) \delta f \cos \left(\omega_{3} t+\theta_{3}\right)\right]\right\}}_{\text {"flux" term }} \underbrace{\left.\sum_{n=1}^{6} \phi_{n}(t)\right)}_{\text {"phase" term }}
$$

Equation (15) is central to this work. It informs us how the SQUID current mixes magnetic flux and junction phase, allowing for gain and dissipation effects at and between different frequencies. In what follows, we treat the response of the SQUID under various, specific pumping conditions. We begin by studying the three-wave degenerate case.

\section{The three-wave degenerate case}

The three-wave degenerate case was treated at length in our previous work [14]. In a threewave parametric amplifier or converter, a pump acts as a source of power to both a signal tone and an idler tone via a nonlinear coupling (e.g., a SQUID). We therefore consider tones at the signal $\left(\omega_{1}\right)$, pump $\left(\omega_{3}\right)$, and idler $\left(\omega_{2}=\omega_{3}-\omega_{1}\right)$ frequencies. Energy conservation in this three-wave case gives $\omega_{1}+\omega_{2}=\omega_{3}$. As we consider this condition to be degenerate, the signal and idler frequencies coincide (i.e., $\omega_{1}=\omega_{2}$ ).

The three-wave degenerate case:

$$
\omega_{1}=\omega_{2}=\left(\omega_{3}-\omega_{1}\right)=\omega_{3} / 2
$$


We use Eq. (15) to determine the response of the SQUID. Since the signal and idler tones are no longer distinct for degenerate conditions, the "phase" term of Eq. (15) simplifies to the following.

$$
\begin{aligned}
\sin [\phi(t)] & \approx \phi(t)=\tilde{\phi}_{1} \cos \left(\omega_{1} t+\theta_{1}\right) \\
& =\frac{1}{2} \tilde{\phi}_{1} e^{j \theta_{p 1}} e^{j \omega_{1} t}+\frac{1}{2} \tilde{\phi}_{1}^{*} e^{-j \theta_{p 1}} e^{-j \omega_{1} t} \quad \text { (degenerate case) }
\end{aligned}
$$

In this section, we depart slightly from the form of Eq. (12) in that we have assumed a cosine dependence with an explicit phase angle. The amplitude $\tilde{\phi}_{1}$ is therefore now real and equal to its complex conjugate $\tilde{\phi}_{1}^{*}$, although we retain the use of conjugate notation for generality.

We did not consider including $\phi_{3}(t)$, which is the junction phase contribution at the pump frequency $\left(\omega_{3}\right)$. This is because we are interested in the signal response. For frequency mixing to occur, components at different frequencies must be multiplied. As long as the approximation $\sin [\phi(t)] \approx \phi(t)$ is valid, $\phi_{3}(t)$ does not contribute to the SQUID current at the signal frequency.

We apply the degenerate condition of Eq. (16) and the "phase" term of Eq. (18) to Eq. (15). From the resulting expression, we find the terms proportionate to the frequency component at $e^{j \omega_{1} t}$. We consider the signal current to be of the form of Eq. (11),

$$
i_{1}(t)=\underbrace{\frac{1}{2} I_{1} e^{j \omega_{1} t}}_{i_{1}(t)_{+}}+\underbrace{\frac{1}{2} I_{1}^{*} e^{-j \omega_{1} t}}_{i_{1}(t)_{-}}
$$

such that we can match its $e^{j \omega_{1} t}$ component, $i_{1}(t)_{+}$, to the following form.

$$
\begin{aligned}
i_{1}(t)_{+} & =\frac{1}{2} I_{1} e^{j \omega_{1} t} \\
& =\frac{1}{2} I_{c} \tilde{\phi}_{1}\left[e^{j \theta_{1}} \cos (F)-\frac{\delta f}{2} \sin (F) e^{j\left(\theta_{3}-\theta_{1}\right)}\right] e^{j \omega_{1} t}
\end{aligned}
$$

Now considering a voltage based on the ac Josephson relation applied to the phase response, we find the following component which is also proportionate to $e^{j \omega_{1} t}$.

$$
\begin{aligned}
v_{1}(t)_{+} & =\frac{1}{2} V_{1} e^{j \omega_{1} t}=\frac{\Phi_{0}}{2 \pi} \frac{d}{d t}\left[\frac{1}{2} \tilde{\phi}_{1} e^{j \theta_{1}} e^{j \omega_{1} t}\right] \\
& =\frac{1}{2}\left[\frac{\Phi_{0} \tilde{\phi}_{1} \omega_{1}}{2 \pi}\left(j e^{j \theta_{1}}\right)\right] e^{j \omega_{1} t}
\end{aligned}
$$

By dividing Eq. (21) by Eq. (22), we can define a signal admittance, $Y\left(\omega_{1}\right)$.

$$
\begin{aligned}
Y\left(\omega_{1}\right) & =\frac{i_{1}(t)_{+}}{v_{1}(t)_{+}} \\
& =\left(j \omega_{1} L_{3 \mathrm{~d}, 0}\right)^{-1}+\left(j \omega_{1} L_{3 \mathrm{~d}, 1}\right)^{-1}
\end{aligned}
$$

Above, we have defined the following identities. 


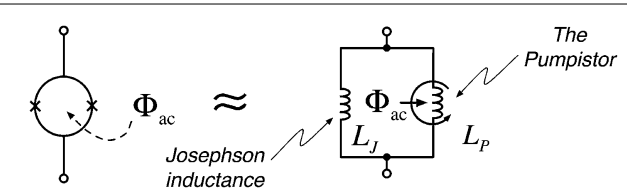

Figure 2 One may solve for the admittance of a flux-modulated SQUID using series expansions for the super-current. The resulting circuit model appears as the Josephson inductance in parallel to a flux- (and phase-) dependent, inductance-like impedance.

Three-wave degenerate amplifier:

$$
\begin{aligned}
& L_{3 \mathrm{~d}, 0}=L_{J} \\
& L_{3 \mathrm{~d}, 1}=-L_{J} \frac{2}{\tan (F)}\left(\frac{1}{\delta f}\right) e^{+j \Delta \theta_{3 \mathrm{~d}}} \\
& \Delta \theta_{3 \mathrm{~d}}=2 \theta_{1}-\theta_{3}
\end{aligned}
$$

The subscript " $3 \mathrm{~d}$ " denotes the three-wave degenerate case. We identify the Josephson inductance, $L_{J}$, from Eq. (6) for the unperturbed flux $\left(\Phi_{\text {ext }}=\Phi_{\mathrm{dc}}\right)$ and small phase $(\phi \approx 0)$ conditions. We therefore consider $L_{J}=\Phi_{0} /\left[2 \pi I_{c} \cos (F)\right]$ for the remainder of this work. From these definitions, Eq. (24) shows that the admittance appears as the parallel combination of the Josephson inductance and a perturbation inductance with an ac-flux dependence (i.e., "the pumpistor" [14]).

Note that this extra inductance, $L_{3 \mathrm{~d}, 1}$, has a dependence on the effective pump phase, $\Delta \theta_{3 \mathrm{~d}}$. Depending on the value of $\Delta \theta_{3 \mathrm{~d}}$, the inductance $L_{3 \mathrm{~d}, 1}$ has both real and imaginary contributions, which may be either positive or negative. Our amplifier topology will be able to supply signal gain when $L_{3 \mathrm{~d}, 1}$ has a substantial negative and real impedance. This depicts the mechanism which allows the SQUID to inject power back into the external circuit at the signal frequency. A diagram of this equivalent circuit is demonstrated in Figure 2.

Here we have treated the degenerate case to first order both in pump flux and in signal phase. We recover the Josephson inductance in combination with a component representing the perturbation to the signal response. This extra impedance, as defined by its frequency dependence, is an inductor. However, its phase dependence allows it to take on complex amplitudes.

It is important to point out that, mathematically, this relation only holds at precisely the frequency $\omega_{1}=\omega_{3} / 2$. When this condition is not met, we need to resort to the general form of the nondegenerate case, which we shall treat in Sections 6 and 7.

Now, we consider some saturation arguments for this three-wave degenerate case.

\subsection{Saturation of the pump flux for the three-wave degenerate case}

As in the theory of mixers [29] and other nonlinear devices, the nonlinear properties of the driven SQUID lead also to saturation effects. These effects include the amplitudedependent modifications of the Josephson inductance, as well as the gain compression of the incremental response.

If we extend the degenerate treatment as in Eq. (23), we can find higher-order parallel inductance terms by expanding the "flux" term of Eq. (14) to higher orders in 
ac flux. Taking the series expansion to third-order, we find the following extension to Eq. (24).

$$
\begin{aligned}
& L_{3 \mathrm{~d}, 0}=L_{J} \\
& L_{3 \mathrm{~d}, 1}=-L_{J} \frac{2}{\tan (F)}\left(\frac{1}{\delta f}\right) e^{+j \Delta \theta_{3 \mathrm{~d}}} \\
& L_{3 \mathrm{~d}, 2}=-4 L_{J}\left(\frac{1}{\delta f}\right)^{2} \\
& L_{3 \mathrm{~d}, 3}=L_{J} \frac{16}{\tan (F)}\left(\frac{1}{\delta f}\right)^{3} e^{+j \Delta \theta_{3 \mathrm{~d}}} \\
& \Delta \theta_{3 \mathrm{~d}}=2 \theta_{1}-\theta_{3}
\end{aligned}
$$

We find that the terms corresponding to the even powers of ac flux contribute to modifying the standard Josephson inductance. Meanwhile, the odd powers modify the phase-dependent term. Knowing that $L_{\mathrm{d}, 1}$ is responsible for gain, we can compare it to its higher-order correction, $L_{\mathrm{d}, 3}$. So by equating $\left|L_{\mathrm{d}, 1}\right|$ to $\left|L_{\mathrm{d}, 3}\right|$ we can estimate the pump acflux amplitude "intercept point." This is only a rough estimate of saturation, and the effects of gain compression would start to become apparent at ac-fluxes considerably smaller than this. To ensure that operation is far from this condition, we would say that the following should always be true.

$$
\frac{\Phi_{\mathrm{ac}}}{\Phi_{0}} \ll \frac{2 \sqrt{2}}{\pi} \approx 0.90
$$

This is not a particularly useful constraint, as we already knew that we wish to keep the total external flux below $\Phi_{0} / 2$. However, we could say that this constraint reinforces the notion that, for properly linearized behavior, $\Phi_{\text {ac }}$ should be maintained at some small fraction of $\Phi_{0}$.

\subsection{Saturation in the signal phase (or voltage) for the three-wave degenerate case}

If we now substitute the phase term of Eq. (15) with an expansion to higher order, we can estimate nonlinear effects due to the magnitude of the signal phase. Here, we assume $\sin (\phi) \approx \phi-\frac{1}{6} \phi^{3}$, with $\phi=\phi_{1}(t)$ from Eq. (18). If we again combine the terms which occur at $e^{j \omega_{1} t}$, we find the 3rd-order correction to the $\Phi_{\text {ac }}$-independent term, $L_{3 \mathrm{~d}, 0}$, to be the following.

$$
1 / L_{3 \mathrm{~d}, 0} \rightarrow 1 / L_{J}\left(1-\frac{\tilde{\phi}_{1}^{2}}{8}\right)
$$

We also find a 3rd-order correction to the $L_{3 \mathrm{~d}, 1}$ inductance term, which was the term inversely proportionate to $\delta f$.

$$
1 / L_{3 \mathrm{~d}, 1} \rightarrow 1 / L_{3 \mathrm{~d}, 1}\left[1+\tilde{\phi}_{1}^{2}\left(\frac{1}{24} e^{j 2 \Delta \theta_{3 \mathrm{~d}}}-\frac{1}{8}\right)\right]
$$

To estimate an "intercept point" due to saturation of the phase amplitude, we can take the maximum of the corrected $1 / L_{3 \mathrm{~d}, 1}$ of Eq. (33), at $\Delta \theta_{3 \mathrm{~d}}=\pi / 2$. It is straightforward to see 
Figure 3 The admittance expansion to higher order, both in external flux and in junction phase, gives a more complete model for the three-wave degenerate case. The even harmonics in the flux expansion serve to only modify the net inductance value. The odd harmonics modify the potential gain and phase-sensitivity. This allows for estimation of the saturation effects. As it is in the theory of mixers, we see in the ac-flux expansion that the third-order term compresses the gain-providing first-order term.

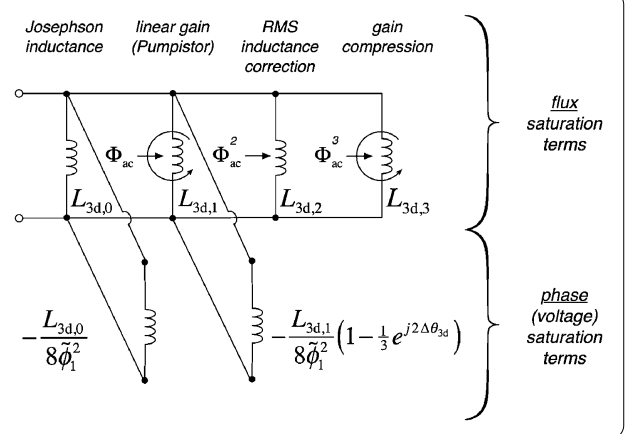

that the contribution of $\tilde{\phi}_{1}^{2}$ should be negligible when the following is true.

$$
\left|\tilde{\phi}_{1}\right| \ll \sqrt{6}
$$

As in the previous consideration of the nonlinearity due to $\Phi_{\mathrm{ac}} / \Phi_{0}$, this is not particularly a remarkable constraint. The phase amplitude $\sqrt{6}$ is obviously already a large fraction of $\pi$. It only reinforces the point that $\left|\tilde{\phi}_{1}\right|$ should be quite small compared to this value. Perhaps, though, it is worthwhile to point out this limit also corresponds directly to a limit on the junction voltage, by way of the ac Josephson effect.

$$
\left|V_{1}\right|=\tilde{\phi}_{1}\left(\frac{\Phi_{0} \omega_{1}}{2 \pi}\right) \ll \frac{\sqrt{2}}{\pi} \Phi_{0} \omega_{1}
$$

Concluding discussion of saturation effects due to flux and to signal phase, we turn to Figure 3. Here, we combine the effects of gain compression into a common model. As in the theory of mixers, we see that the odd terms in the expansion account for both gain and its saturation.

\section{The four-wave degenerate case}

Next, we take interest in the SQUID with zero dc flux. When the dc flux is zero, the first derivative of inductance as a function of flux is also zero. We notice from Eq. (27) that $L_{3 \mathrm{~d}, 1}$ becomes infinite (an "open") and no longer contributes to the circuit. In fact, all of the odd powers of $\Phi_{\mathrm{ac}}$ will disappear from the "flux" term of Eq. (15). The reason for this can be attributed to the symmetric behavior of the unbiased device. Yet it is still possible to achieve parametric amplification among the even harmonics of the admittance expansion in flux, in a degenerate case without an idler tone distinct from a signal $\left(\omega_{1}=\omega_{2}\right)$. In this case one must use four-wave degenerate mixing, where we can consider this as two pump photons interacting with a signal photon and an idler photon (i.e., $\omega_{1}+\omega_{2}=2 \omega_{3}$ ).

The four-wave degenerate case:

$$
\omega_{1}=\omega_{2}=\left(2 \omega_{3}-\omega_{1}\right)=\omega_{3}
$$

As in the three-wave degenerate case, both idler and signal tones occur at identically the same frequency and we consider only the disturbance of their combined response. Again, we treat this degenerate tone as the signal $\left(\omega_{1}\right)$ response.

When the external magnetic flux is comprised of solely the ac component, we mentioned that the device behaves symmetrically around zero flux. To find the relevant dynamical 
response, we need to expand the "flux" term of Eq. (14) to 2nd-order for this zero flux-bias case.

$$
\begin{aligned}
I_{c} \cos \left(\pi \delta \Phi / \Phi_{0}\right) & \approx I_{c}-I_{c} \frac{\pi^{2}}{2 \Phi_{0}^{2}}(\delta \Phi)^{2} \\
& =I_{c}-\frac{I_{c}}{2}(\delta f)^{2}\left[\cos \left(\omega_{3} t+\theta_{3}\right)\right]^{2}
\end{aligned}
$$

As in Eq. (15), we find the total current at the signal frequency by multiplying our "flux" approximation by the "phase" term approximation. We use the approximation for the signal phase as in Eq. (18). The resulting signal current, analogous to Eq. (21) but with $\omega_{1}=\omega_{3}$, becomes

$$
i_{1}(t)_{+}=\frac{1}{2} I_{c} \tilde{\phi}_{1}\left[1-\frac{1}{4}(\delta f)^{2}\right] e^{j \theta_{1}} e^{j \omega_{1} t}-\frac{1}{16} I_{c} \tilde{\phi}_{1}^{*}(\delta f)^{2} e^{j 2 \theta_{3}-j \theta_{1}} e^{j \omega_{1} t}
$$

Considering the small-signal voltage of Eq. (22), we find the signal admittance in the four-wave degenerate case to be

$$
\begin{aligned}
Y_{4 \mathrm{~d}}\left(\omega_{1}\right) & =\frac{2 I_{c} \pi}{j \omega_{1} \Phi_{0}}-\frac{I_{c} \pi}{j 2 \omega_{1} \Phi_{0}}(\delta f)^{2}-\frac{I_{c} \pi}{j 4 \omega_{1} \Phi_{0}}(\delta f)^{2} e^{j 2 \theta_{3}-j 2 \theta_{1}} \\
& =\left(j \omega_{1} L_{4 \mathrm{~d}, 0}\right)^{-1}+\left(j \omega_{1} L_{4 \mathrm{~d}, 2 \mathrm{a}}\right)^{-1}+\left(j \omega_{1} L_{4 \mathrm{~d}, 2 \mathrm{~b}}\right)^{-1}
\end{aligned}
$$

In this case, we have defined the following.

Four-wave degenerate amplifier:

$$
\begin{aligned}
& L_{4 \mathrm{~d}, 0}=L_{J} \\
& L_{4 \mathrm{~d}, 2 \mathrm{a}}=-4 L_{J}\left(\frac{1}{\delta f}\right)^{2} \\
& L_{4 \mathrm{~d}, 2 \mathrm{~b}}=-8 L_{J}\left(\frac{1}{\delta f}\right)^{2} e^{j \Delta \theta_{4 \mathrm{~d}}} \\
& \Delta \theta_{4 \mathrm{~d}}=2\left(\theta_{1}-\theta_{3}\right)
\end{aligned}
$$

So we find that the admittance which is proportionate to $(\delta f)^{2}$ has both phase-insensitive and phase-sensitive terms. Note also the dependence on the pump phase in $\Delta \theta_{4 \mathrm{~d}}$ is different by 2 compared to the phase angle $\Delta \theta_{3 \mathrm{~d}}$ of Eq. (25). Also in this four-wave degenerate case, we can produce a negative resistance, and consequently gain, from the $L_{4 \mathrm{~d}, 2 \mathrm{~b}}$ term by adjusting $\Delta \theta_{4 \mathrm{~d}}$ accordingly.

In the next sections, we turn to the more general case of nondegenerate operation. There, the idler response must now be considered separately from the signal response.

\section{General conditions for nondegenerate parametric effects using the small-signal admittance matrix}

We now consider specifically nondegenerate mixing conditions. Here, "nondegenerate" asserts its standard meaning that all frequency terms under consideration are unique, i.e., $\omega_{i} \neq \omega_{j}$ for all $j \neq i$. Where any of our six considered mixing frequencies (Table 1 ) may contribute to a flux-pumped SQUID circuit, we work within our typical small-signal limit using a linearized system of equations. From this, we will develop an equivalent admittance matrix. 
As before, the SQUID current is directly related to the junction phase by the dc Josephson effect as in Eq. (15). However, we include a 2nd-order expansion of the "flux" term of Eq. (14), which also includes dc flux. In this case, expanding the "flux" term to 2ndorder ensures nontrivial couplings to most frequency components. We wish to find the contributions of the current at different frequencies, given by the form $i(t)=\sum_{n=1}^{6} i_{n}(t)$ as in Eq. (11). For a single frequency component of the junction phase, we find the current amplitudes at all considered frequencies.

Although we could present a matrix of frequency couplings by what we have just described, we wish to find an admittance matrix relating current and voltage amplitudes. We therefore translate junction phase amplitudes into voltage amplitudes by way of the ac Josephson effect. Starting from Eq. (10), we find the following.

$$
\phi_{n}(t)=\frac{2 \pi}{\Phi_{0}} \int v_{n}(t) d t=-j \frac{\pi}{\Phi_{0} \omega_{n}} V_{n} e^{j \omega_{n} t}+j \frac{\pi}{\Phi_{0} \omega_{n}} V_{n}^{*} e^{-j \omega_{n} t}
$$

Taking into consideration how frequency components of the voltage couple to both conjugate and non-conjugate terms of the current, we arrive at our desired small-signal admittance matrix. Rather than a basis set of physical ports as in a multi-terminal device, here the admittance matrix "ports" (indices) represent the frequencies from Table 1.

$$
\left(\begin{array}{c}
I_{1} \\
I_{2}^{*} \\
I_{4} \\
I_{5}^{*} \\
I_{6}
\end{array}\right)=\frac{1}{j L_{J}}\left(\begin{array}{ccccc}
\frac{\epsilon_{0}}{\omega_{1}} & -\frac{\epsilon_{1}^{*}}{\omega_{2}} & \frac{\epsilon_{1}}{\omega_{4}} & -\frac{\epsilon_{2}^{*}}{\omega_{5}} & \frac{\epsilon_{2}}{\omega_{6}} \\
\frac{\epsilon_{1}}{\omega_{1}} & -\frac{\epsilon_{0}}{\omega_{2}} & \frac{\epsilon_{2}}{\omega_{4}} & -\frac{\epsilon_{1}^{*}}{\omega_{1}} & 0 \\
\frac{\epsilon_{1}^{*}}{\omega_{1}} & -\frac{\epsilon_{2}^{*}}{\omega_{2}} & \frac{\epsilon_{0}}{\omega_{4}} & 0 & \frac{\epsilon_{1}}{\omega_{6}} \\
\frac{\epsilon_{2}}{\omega_{1}} & -\frac{\epsilon_{1}}{\omega_{2}} & 0 & -\frac{\epsilon_{0}}{\omega_{5}} & 0 \\
\frac{\epsilon_{2}^{*}}{\omega_{1}} & 0 & \frac{\epsilon_{1}^{*}}{\omega_{4}} & 0 & \frac{\epsilon_{0}}{\omega_{6}}
\end{array}\right)\left(\begin{array}{c}
V_{1} \\
V_{2}^{*} \\
V_{4} \\
V_{5}^{*} \\
V_{6}
\end{array}\right)
$$

We do not list in this matrix the pump current amplitude, $I_{3}$, as it couples to none of the other six frequencies but its own $\left(\omega_{3}\right)$.

This admittance matrix holds true as long as the pump frequency is larger than the signal frequency $\left(\omega_{3}>\omega_{1}\right)$ so that the "three-wave difference idler" frequency remains positive $\left(\omega_{2}>0\right)$. In the case of $\omega_{3}<\omega_{1}$, some matrix elements appear instead with conjugate quantities. Similar redefinitions are also necessary if frequency $\omega_{5}=2 \omega_{3}-\omega_{1}$ were also to become negative. We consider the conditions $\left(\omega_{2}>0\right)$ and $\left(\omega_{5}>0\right)$ to be the standard situation.

We find again the quiescent Josephson inductance, $L_{J}=\frac{\Phi_{0}}{2 \pi I_{c} \cos (F)}$. Some new, fluxdependent terms $\epsilon_{0}, \epsilon_{1}$, and $\epsilon_{2}$ also appear, which are not indexed by frequency. Rather, their indices indicate the order of the series expansion in flux for which they first become nontrivial. Their expressions are the following.

$$
\begin{aligned}
& \epsilon_{0}=1-\frac{1}{4} \delta f^{2} \\
& \epsilon_{1}=\frac{\delta f}{2} \tan (F) e^{-j \theta_{3}} \\
& \epsilon_{2}=\frac{\delta f^{2}}{8} e^{-j 2 \theta_{3}}
\end{aligned}
$$

To note, for vanishing $\delta f=\frac{\pi \Phi_{\mathrm{ac}}}{\Phi_{0}}$, the limit of $\epsilon_{0}$ is unity, while $\epsilon_{1}$ and $\epsilon_{2}$ tend to zero. 
The importance of the matrix equation (Eq. (43)) should be emphasized. This tells us the response of a flux-pumped SQUID between all relevant frequency components, but yet it can be used in the same form as any other n-port admittance matrix from circuit theory. So for this very general degenerate case, we may now consider a large number of three-wave and four-wave mixing devices, both as negative-resistance amplifiers and as frequency converters. It further allows us to describe a number of next-order effects which also occur in these devices.

The elements of the admittance matrix (Eq. (43)), are specifically the short-circuit admittance parameters [30]. This is defined as the following,

$$
Y_{k l}=\left.\frac{I_{k}}{V_{l}}\right|_{V_{m}=0, m \neq l}
$$

where $V_{m}=0$ with $m \neq l$ is a condition met by shorting all ports, $m$ other than the port of interest, $l$.

In the next section, we begin by considering a special case of Eq. (43) where the desired harmonics form a subset of the admittance matrix. The unwanted components are assumed to be zero (i.e., shorted). We will then find necessary corrections for when unwanted harmonics are instead open-circuited.

\section{The three-wave nondegenerate negative-resistance parametric amplifier}

When the signal frequency under consideration is not degenerate relative to the pump frequency, the findings of Sections 4 and 5 break down. We now return to considerations of three-wave mixing, but for the nondegenerate case where $\omega_{1} \neq \omega_{3} / 2$. In this case, it is necessary to provide for the presence of an idler junction phase (voltage) at $\omega_{2}$. The idler comes about due to the nonlinear frequency coupling between the signal and pump terms. A response at the idler frequency need not be induced at the input, nor measured as an output variable, for it to play an important role as an internal state variable.

In this section, we consider the following conditions on the signal and idler frequencies.

The three-wave nondegenerate case:

$$
\omega_{2}=\left(\omega_{3}-\omega_{1}\right) \neq \omega_{1}
$$

We consider the matrix subset of Eq. (43) corresponding to a signal at $\omega_{1}$ and the idler at $\omega_{2}$. The circuit at all other harmonics is assumed to be shorted.

$$
\left(\begin{array}{l}
I_{1} \\
I_{2}^{*}
\end{array}\right)=\frac{1}{j L_{J}}\left(\begin{array}{ll}
\frac{\epsilon_{0}}{\omega_{1}} & -\frac{\epsilon_{1}^{*}}{\omega_{2}} \\
\frac{\epsilon_{1}}{\omega_{1}} & -\frac{\epsilon_{0}}{\omega_{2}}
\end{array}\right)\left(\begin{array}{c}
V_{1} \\
V_{2}^{*}
\end{array}\right)
$$

This provides the current and voltage relations directly across the SQUID at the signal and idler frequencies. Next, we generalize the circuit such that we take into account the possible effects of other generator and load admittances.

\subsection{Understanding this three-wave nondegenerate model as a circuit topology}

To conceptualize the system we have just described, consider the flux-pumped SQUID as the primary element of a multi-frequency circuit. This is depicted in Figure 4(a). We assume this circuit to be sourced by a signal current $i_{s}(t)$ of the form of Eq. (11), such that 
Figure 4 Considerations of the external circuit for the three-wave nondegenerate case. (a) This figure depicts the physical, general circuit considered in this section, containing a flux-pumped SQUID. This circuit accounts for an external source current, $i_{s}$, as well as external $\left(Y_{\text {ext }}\right)$ and local shunt $\left(Y_{\text {sh }}\right)$ admittances. (b) It is possible to represent the general circuit in an equivalent way that separates the external loading effects of the circuit at the "signal" $\left(\omega_{1}\right)$ and "idler" $\left(\omega_{2}\right)$ frequencies. This is done by introducing hypothetical, ideal bandpass filters at $\omega_{1}$ and $\omega_{2}$. These filters act open-circuited at their respective frequency, but short-circuited for all other frequencies. In this representation, the external admittance, $Y_{\text {ext, }}$ is represented at different frequencies by $Y_{1}$ and $Y_{2}$. The input admittance $\left(Y_{\mathrm{SQ}}\right)$ is then based on the signal voltage response to current $i_{1}$, which depends upon the idler in a way that may allow for gain.

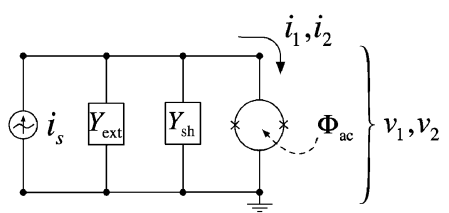

(a)

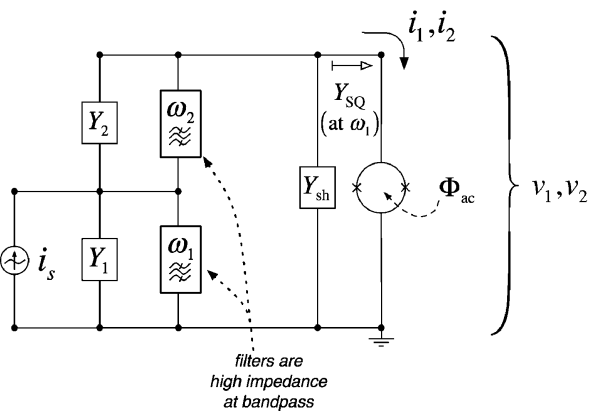

(b)

its amplitude is $I_{s}$ and its frequency is $\omega_{1}$. This external current source may be loaded by an external admittance, $Y_{\text {ext }}$. The currents $i_{1}(t)$ and $i_{2}(t)$, with amplitudes $I_{1}$ and $I_{2}$, continue to indicate the currents directly into the SQUID at frequencies $\omega_{1}$ and $\omega_{2}$, respectively. We account for either parasitic or intentional admittances directly across the SQUID by the term $Y_{\mathrm{sh}}(\omega)$, which may be frequency dependent. We make a distinction between $Y_{\mathrm{ext}}$ and $Y_{\mathrm{sh}}(\omega)$ since the definition of available power from the external source involves only $\operatorname{Re}\left[Y_{\text {ext }}\right]$.

In Figure 4(b), we depict how we can think of the effects of the external load at different frequencies by recasting this circuit in an equivalent representation. In this case, we separate $Y_{\text {ext }}$ into distinct impedances $Y_{1}$ at frequency $\omega_{1}$ and $Y_{2}$ at frequency $\omega_{2}$. We introduce hypothetical bandpass filters which isolate $Y_{1}$ and $Y_{2}$ to their respective frequencies outside of the pumped SQUID. These ideal filters work by providing a high-impedance (open) at their intended frequencies, while at all other frequencies they serve as a perfect short. This topology ensures that all unwanted frequencies short the SQUID, preventing any voltage at those frequencies to accumulate. Thus, we are able to reduce the general admittance matrix of Eq. (43) to the much simpler matrix of Eq. (49). While we do not actively source the idler current, we will find that the external admittance at the idler frequency, $Y_{2}$, effects response of the SQUID at the signal frequency in an important way.

\subsection{The voltage and current ratios of the three-wave nondegenerate parametric amplifier}

We are not quite ready to understand how gain appears in this system. This nondegenerate case is complicated by the appearance of an idler response distinct from the signal. For instance, the idler-to-signal voltage ratio $\frac{V_{2}^{*}}{V_{1}}$ will become important. To find a relation for $V_{2}^{*}$, we examine the second line of the matrix equation (49). While it is clear that we need to solve for $V_{2}^{*}$, what is $I_{2}^{*}$ ? Unlike the signal response, we are not sourcing or measuring an idler current. The idler current is the result of voltage disturbances at the idler frequency, coupled to the external circuit of the surrounding electrical system. Consequently, we must specify how the circuit is loaded at the idler frequency. This is why specifying some external (conjugate) idler admittance, $Y_{2}^{*}$, was necessary in the previous 
section. In what follows, we complete an analysis of our generalized circuit to solve for the idler voltage and current in terms of the signal.

Regarding the general circuit as depicted in Figure 4, we use Kirchhoff's node equations for both the signal and idler.

$$
\begin{aligned}
& I_{s}-V_{1} Y_{1}^{\prime}-I_{1}=0 \\
& -V_{2}^{*} Y_{2}^{*}-I_{2}^{*}=0
\end{aligned}
$$

Above, we defined the grouped admittances $Y_{1}^{\prime}=Y_{1}+Y_{\mathrm{sh}}\left(\omega_{1}\right)$ and $Y_{2}^{\prime}=Y_{2}+Y_{\mathrm{sh}}\left(\omega_{2}\right)$. To go further, the coupled subsystem of Eq. (49) allows us to eliminate $I_{1}$ and $I_{2}^{*}$, giving the following.

$$
\begin{aligned}
& I_{s}=V_{1}\left(Y_{1}^{\prime}+\frac{\epsilon_{0}}{j \omega_{1} L_{J}}\right)-\frac{V_{2}^{*} \epsilon_{1}^{*}}{j \omega_{2} L_{J}} \\
& 0=\frac{V_{1} \epsilon_{1}}{j \omega_{1} L_{J}}+V_{2}^{*}\left(Y_{2}^{\prime *}-\frac{\epsilon_{0}}{j \omega_{2} L_{J}}\right)
\end{aligned}
$$

Equations (52) and (53) now represent the current and voltage response of the generalized circuit depicted in Figure 4. Since the signal current is sourced in this model, what remains to be solved are the voltage disturbances $V_{1}$ and $V_{2}^{*}$. We define the impedances $Z_{L 1}=$ $j \omega_{1} L_{J} / \epsilon_{0}$ and $Z_{L 2}=j \omega_{2} L_{J} / \epsilon_{0}$. The voltage amplitudes are then found to be

$$
\begin{aligned}
& V_{1}=L_{J}^{2} Z_{L 1} \omega_{1} \omega_{2}\left(Y_{2}^{\prime *} Z_{L 2}-1\right)\left(\frac{I_{s}}{\Delta}\right) \\
& V_{2}^{*}=j L_{J} Z_{L 1} Z_{L 2} \epsilon_{1} \omega_{2}\left(\frac{I_{s}}{\Delta}\right)
\end{aligned}
$$

where the denominator term, $\Delta$, is proportionate to the determinant formed by the matrix of Eqs. (52) and (53).

$$
\Delta=L_{J}^{2} \omega_{1} \omega_{2}\left(Y_{1} Z_{L 1}+1\right)\left(Y_{2}^{\prime *} Z_{L 2}-1\right)-Z_{L 1} Z_{L 2}\left|\epsilon_{1}\right|^{2}
$$

When we consider the voltage ratio between the idler and signal, the cumbersome denominator cancels, providing the more simple relation

$$
\frac{V_{2}^{*}}{V_{1}}=\frac{\omega_{2} \epsilon_{1}}{\omega_{1} \epsilon_{0}} \frac{1}{1+Z_{L 2}^{*} Y_{2}^{\prime *}}
$$

Here $Z_{L 2}^{*} Y_{2}^{\prime *} \ll 1$, we see Eq. (57) go to the limit

$$
\lim _{Z_{L 2}^{*} Y_{2}^{* *} \rightarrow 0}\left(\frac{V_{2}^{*}}{V_{1}}\right)=\frac{\omega_{2} \epsilon_{1}}{\omega_{1} \epsilon_{0}}=\frac{\omega_{2}}{2 \omega_{1}} \frac{\delta f}{1-\frac{1}{4} \delta f^{2}} \tan (F) e^{-j \theta_{3}}
$$

On the other hand, when this quantity becomes large such that $Z_{L 2}^{*} Y_{2}^{*} \gg 1$, we see

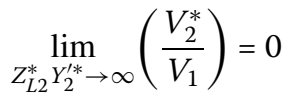


So the voltage of the idler response is of course a function of how well the external circuit is being kept "open" at the idler frequency, $\omega_{2}$.

We can also find the idler-to-signal current ratio. For this we revisit the system represented by Eq. (49), and divide its second equation by its first. We substitute the signal and idler voltage amplitudes found in Eqs. (54) and (55). This gives the following.

$$
\frac{I_{2}^{*}}{I_{1}}=\frac{\epsilon_{1}}{\epsilon_{0}} \frac{1}{1+\frac{1}{Z_{L 2}^{*} Y_{2}^{1 *}}+\frac{\left|\epsilon_{1}\right|^{2}}{\epsilon_{0}^{2}} \frac{\omega_{1}}{\omega_{2} Z_{L 1} Y_{2}^{\prime *}}}
$$

We can look at the admittance limits of the current ratio as well. When the external admittance is small, we see

$$
\lim _{Z_{L 2}^{*} Y_{2}^{* *} \rightarrow 0}\left(\frac{I_{2}^{*}}{I_{1}}\right)=0
$$

Conversely, when external admittance is large, we see

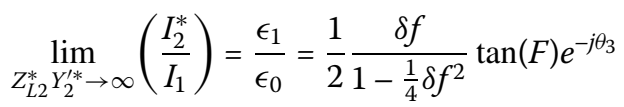

These limits are intuitive. We can see the idler current will be inhibited when the external circuit is comparatively more "open," representing a small external admittance. Note the similar behavior indicated between Eqs. (58) and (61), as well as between Eqs. (59) and (62).

These quantities depict the response of the circuit at the idler frequency, $\omega_{2}$, relative to the circuit behavior at the signal frequency, $\omega_{1}$. We will now utilize this understanding in the next section to find how this system acts as a negative-resistance amplifier.

\subsection{The input impedance of the nondegenerate three-wave parametric amplifier}

To understand how this system works as an amplifier, we must find how it provides a negative resistance at the signal frequency. To this end, we seek to find the input admittance as seen at $\omega_{1}$.

The input admittance as seen into the device at the signal frequency we can say is $Y_{\mathrm{SQ}}=$ $I_{1} V_{1}$, giving

$$
Y_{\mathrm{SQ}}=\frac{I_{1}}{V_{1}}=\frac{1}{Z_{L 1}}-\frac{V_{2}^{*}}{V_{1}} \frac{\epsilon_{1}^{*}}{\epsilon_{0}} \frac{1}{Z_{L 1}}
$$

Recall that $\epsilon_{0} \approx 1$ to first order. To interpret Eq. (63) as an impedance, this is the Josephson inductance again in parallel to some other term. To find this other term, which is represented (as an admittance) by the second term on the right-hand side of Eq. (63), we must incorporate the ratio $\frac{V_{2}^{*}}{V_{1}}$ from Eq. (57). Substituting this term into Eq. (63), we arrive at

$$
\begin{aligned}
Y_{\mathrm{SQ}} & =\frac{1}{Z_{L 1}}-\frac{\left|\epsilon_{1}\right|^{2}}{\epsilon_{0}^{2}} \frac{1}{Z_{L 1}} \frac{1}{1+Z_{L 2}^{*} Y_{2}^{* *}} \\
& =\left(j \omega_{1} L_{\mathrm{n}, 0}\right)^{-1}+\left(j \omega_{1} L_{\mathrm{n}, 2}\right)^{-1}
\end{aligned}
$$

We have therefore represented the input admittance again as inductive terms. We determined a parallel inductance model before, in the degenerate case, but here the dependence 


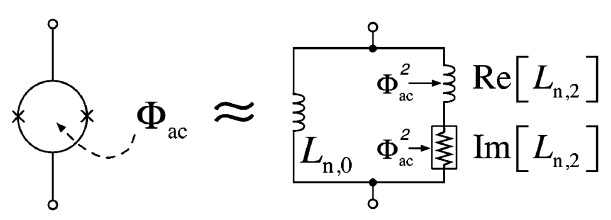

Figure 5 This figure depicts the equivalent signal impedance of the flux-pumped SQUID in the three-wave nondegenerate case. The constituent inductances are given by Eq. (66). In the limit that the ac-flux is small such that $\gamma_{0} \approx 1$, the inductance $L_{n, 0}$ is simply the quiescent Josephson inductance and $L_{\mathrm{n}, 2} \propto \Phi_{\mathrm{ac}}^{2}$. The $L_{\mathrm{n}, 2}$ acquires an imaginary component due to the external (real) admittance at the idler frequency. A positive, imaginary inductance is a negative, real impedance, which may therefore provide gain.

on the pump phase is no longer present. This nondegenerate amplifier, therefore, is phase insensitive. The following terms for inductances are used.

Three-wave nondegenerate amplifier:

$$
\begin{aligned}
& L_{\mathrm{n}, 0}=L_{J} / \varepsilon_{0} \\
& L_{\mathrm{n}, 2}=-\frac{L_{J}}{\left|\varepsilon_{1}\right|^{2}}\left(\varepsilon_{0}-j \omega_{2} L_{J} Y_{2}^{\prime *}\right)
\end{aligned}
$$

Above, the " $L_{\mathrm{n}, 0}$ " inductance is once again simply the Josephson inductance in the smallsignal limit. The " $L_{\mathrm{n}, 2}$ " inductance, in parallel to $L_{\mathrm{n}, 0}$, contains two terms which are both proportionate to $\left|\epsilon_{1}\right|^{-2}$. The first term is negative and simply modifies the net inductance by a small correction, making the net inductance appear bigger as the ac flux increases. The second term of $L_{\mathrm{n}, 2}$ depends on $Y_{2}^{*}$ in an important way, providing the possibility for gain in this scenario. If $Y_{2}^{\prime *}$ has a real and positive component, this allows the impedance represented by $L_{\mathrm{n}, 2}$ to acquire a negative and real component. Therefore the $Y_{2}^{\prime *}$ term of $L_{\mathrm{n}, 2}$ acts as an active impedance converter, allowing the impedance external to the SQUID at the idler frequency to appear, transformed, at the signal frequency. We may think of the input admittance (or input impedance $Z_{\mathrm{SQ}}=1 / Y_{\mathrm{SQ}}$ ) directly into the three-wave nondegenerate pumped SQUID as depicted in Figure 5.

We comment on the frequency dependence of $L_{\mathrm{n}, 2}$. If we subscribe to axiomatic circuit theory [31-33], our linearized inductances should have a dependence strictly proportional to $j \omega$. The second term in $L_{\mathrm{n}, 2}$, which is the same term that may act as a negative resistance, also contains an extra factor, $j \omega_{2}=j\left(\omega_{3}-\omega_{1}\right)$. This gives a maximum of the product $\omega_{1} \omega_{2}$ at $\omega_{3} / 2$, which for this reason is why $\omega_{3} / 2$ is the frequency of maximum parametric amplification (or nearly so) in a three-wave nondegenerate amplifier. Between an uncommon frequency dependence and negative-resistance behavior, it may be logical to consider this second term of $L_{\mathrm{n}, 2}$ as relating to something other than an inductance. Yet we choose keep the terminology of an inductance only for consistency.

To conclude this section, we repeat that we have found the negative resistance that provides gain in this three-wave nondegenerate amplifier. This appears in the imaginary component of the term $L_{\mathrm{n}, 2}$ from Eq. (66). Although the frequency mixing between the idler and signal is provided for by the pump, the negative resistance occurs as an effect of mapping the idler's external (real) load admittance back onto the signal as a negative resistance. 


\subsection{The three-wave nondegenerate amplifier: transducer gain}

The common readout implementation for a parametric amplifier (e.g., a flux-pumped SQUID) is as a reflection device coupled to a circulator and a 2nd-stage amplifier (e.g., a high electron mobility transistor (HEMT)) [7, 9, 14, 22-24]. It is therefore important that the first-stage gain of a parametrically flux-pumped SQUID be adequate to overcome the noise of subsequent gain stages. An insightful quantity in this context (in addition, say, to other quantities such as a noise figure) is the transducer gain of the device. It is straightforward to specify the transducer gain, considering the simplified circuit we have so far described in this section.

The transducer gain is the ratio of the output power to the available input power. We consider the source admittance as $Y_{1}$. For the (rms) available input power at the signal frequency, we find

$$
P_{\mathrm{a}, 1}=\frac{I_{s}^{2}}{8 \operatorname{Re}\left[Y_{1}\right]}
$$

We consider the output signal to be reflected back onto the input admittance, such that we say the (rms) output power is

$$
P_{\mathrm{o}, 1}=\frac{V_{1}^{2}}{2} \operatorname{Re}\left[Y_{1}\right]
$$

The transducer gain is then

$$
G_{T}=\frac{P_{\mathrm{o}, 1}}{P_{\mathrm{a}, 1}}=\frac{4 V_{1}^{2}\left(\operatorname{Re}\left[Y_{1}\right]\right)^{2}}{I_{s}^{2}}=4 \frac{\left(\operatorname{Re}\left[Y_{1}\right]\right)^{2}}{\left|Y_{1}^{\prime}+Y_{\mathrm{SQ}}\right|^{2}}
$$

This can be expressed as

$$
G_{T}=\frac{4 \operatorname{Re}\left[Y_{1}\right]^{2}}{\left(\frac{1}{\omega_{1} L_{\mathrm{n}, 0}}+\frac{\operatorname{Re}\left[L_{\mathrm{n}, 2}\right]}{\omega_{1}\left|L_{\mathrm{n}, 2}\right|^{2}}-\operatorname{Im}\left[Y_{1}^{\prime}\right]\right)^{2}+\left(\frac{\operatorname{Im}\left[L_{\mathrm{n}, 2}\right]}{\omega_{1}\left|L_{\mathrm{n}, 2}\right|^{2}}-\operatorname{Re}\left[Y_{1}^{\prime}\right]\right)^{2}}
$$

where $L_{\mathrm{n}, 0}$ and $L_{\mathrm{n}, 2}$ are from Eq. (66).

\subsection{Adding open-circuited terms}

As an admittance model, as opposed to an impedance model, the ideal case is for all non-intentional harmonics to be subject to an infinite admittance external to the pumped SQUID (e.g., to have a shorted external load at frequencies other than the signal and idler). This prevents voltages at these other frequencies from accumulating across the SQUID, thereby removing their influence from the admittance matrix and the resulting mixed currents. Conversely, when the external impedance is nontrivial at other frequencies, other harmonics will modify the description we have just presented.

Here, we treat the case opposite from before, where we now consider frequencies other than $\omega_{1}$ and $\omega_{2}$ to be open-circuited. Therefore, we consider the last three rows of the admittance matrix of Eq. (43) to represent no current flow, setting currents $I_{4}, I_{5}^{*}$, and $I_{6}$ to zero. We solve for the voltage amplitudes of these harmonics, which are now nontrivial. We substitute these voltage amplitudes into the expressions for current at the signal $\left(\omega_{1}\right)$ 


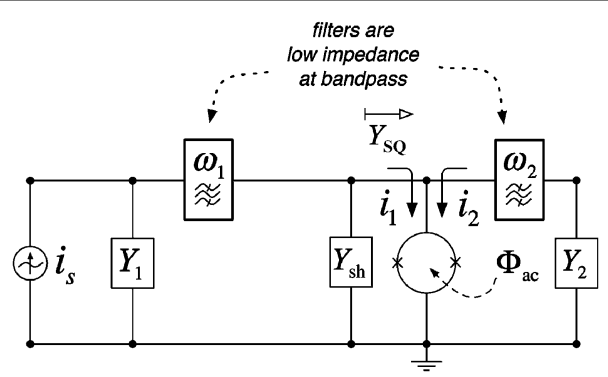

Figure 6 This figure demonstrates the parametric interaction of the open-circuited SQUID at the signal and idler frequencies. As opposed to Section 7.1, these series bandpass filters are now zero impedance at bandpass and blocking at all other frequencies. This works in such a way that frequencies other than $\omega_{1}$ and $\omega_{2}$ now present an open circuit to the pumped SQUID. Therefore the voltage across the SQUID is not necessarily zero at these other frequencies. These additional mixing effects can be mapped onto a modified subsystem between signal and idler, which is the $2 \times 2$ matrix of Eq. (71).

and idler $\left(\omega_{2}\right)$ frequencies. To reach a manageable solution, we assume the limiting conditions $\epsilon_{0} \approx 1$ and $\epsilon_{2} \approx 0$ for currents at $\omega_{4}, \omega_{5}$, and $\omega_{6}$. If we keep terms up to $\delta f^{2}$, we find the signal-idler subset matrix has the simple form,

$$
\left(\begin{array}{l}
I_{1} \\
I_{2}^{*}
\end{array}\right)=\frac{1}{j L_{J}}\left(\begin{array}{cc}
\frac{\varepsilon_{0}}{\omega_{1}}\left[1-\frac{\left|\varepsilon_{1}\right|^{2}}{\varepsilon_{0}}\right] & -\frac{\varepsilon_{1}^{*}}{\omega_{2}} \\
\frac{\varepsilon_{1}}{\omega_{1}} & -\frac{\varepsilon_{0}}{\omega_{2}}\left[1-\frac{\left|\varepsilon_{1}\right|^{2}}{\varepsilon_{0}}\right]
\end{array}\right)\left(\begin{array}{c}
V_{1} \\
V_{2}^{*}
\end{array}\right)
$$

We find this system identical to that of Eq. (49), except for the multiplicative correction factor in square brackets, $\left[1-\frac{\left|\varepsilon_{1}\right|^{2}}{\varepsilon_{0}}\right]$, appearing in the two matrix elements of the main diagonal. This correction factor may become significant even for reasonably small $\delta f$ as the dc flux, $F$, approaches $\pi / 2$. This is the notable difference between this open-circuited case and the short-circuited case we treated in Section 7.3.

We illustrate the open-circuited case as an equivalent circuit in Figure 6. We depict signal and idler circuits now directly in parallel to the pumped SQUID. As opposed to the short-circuited case depicted in Figure 4(b), here the ideal filters are accomplished in series such that only the permitted frequency is allowed to pass, while all other frequencies see an open-circuit.

In this section, we have determined the response of the three-wave nondegenerate amplifier as an impedance model. This is analogous to the "pumpistor" models we found for the three-wave and four-wave degenerate cases treated in Sections 4 and 5. A notable difference in this nondegenerate case is that the external admittance at the idler frequency now determines the negative resistance. As can be seen by Eq. (66), for a negative resistance to occur at the signal frequency, it is necessary that the circuit external to the SQUID at the idler frequency appear as a positive and real admittance. By treating both a "short-circuited" and an "open-circuited" model, we found that a finite external admittance at harmonics other than the signal and idler frequencies may also affect amplifier performance.

\section{Conclusions}

In conclusion, we have substantially extended the equivalent impedance models of a fluxpumped SQUID which we first put forth for the three-wave degenerate case [14]. For all 
general classes of parametric driving, a flux-pumped SQUID can be described at the signal frequency as a Josephson inductance in parallel to an effective, flux-dependent circuit element, "the pumpistor." Parametric amplification can be intuitively understood within this framework, as the pumpistor impedance manifests in whole or in part as a negative resistance.

We reviewed three-wave degenerate pumping, which explains why gain in this case should be phase sensitive between the signal and pump. For this case, we also extended our impedance approximation to demonstrate how the SQUID saturates both by pump flux and by junction phase (or voltage). We also depicted the four-wave degenerate case which is appropriate when the device is biased with zero-flux. Here, the pumpistor element is inversely proportionate to the square of the ac flux. We found this case also to be phase sensitive, but with a slightly different signal-to-pump difference than in the threewave degenerate case.

We also depicted nondegenerate pumping in a very general sense, using a matrix equation formalism. This formalism accounts for the presence of one or up to four "idler" frequencies which occur as mixing tones between the pump and the signal response. Many three- and four-wave nondegenerate parametric phenomena can be interpreted from this matrix, including effects such as frequency up- and down-conversion. Using a subset of these matrix equations, we treated the three-wave nondegenerate amplifier, where the signal and single idler are considered. By solving for an idler distinct from the signal, we found that the pumpistor impedance was now phase insensitive. We found the negative resistance responsible for gain was now dependent on the external circuit admittance at the idler frequency. With regards to the other, higher harmonics, we treated the threewave nondegenerate amplifier in both the "short-circuited" and "open-circuited" approximations. While all of these models operate under a classical, circuit-theoretic framework rather than a quantum optics framework, they should be of great benefit for future designs of experiments using superconducting circuits for quantum information purposes.

Competing interests

The authors declare that they have no competing interests.

Authors' contributions

KMS derived most of the equations. Both authors developed the concept and wrote this paper together.

\section{Author details}

${ }^{1}$ Electrical and Computer Engineering, Texas A\&M University, College Station, TX 77843, USA. ${ }^{2}$ Microtechnology and Nanoscience, Chalmers University of Technology, Göteborg, SE-412 96, Sweden.

\section{Acknowledgements}

We acknowledge support from the EU through the ERC and the projects SOLID, SCALEQIT, and PROMISCE, as well as from the Swedish Research Council and the Wallenberg Foundation. We are also grateful for fruitful discussions with Chris Wilson, Seckin Kintaş, Michaël Simoen, Philip Krantz, Martin Sandberg, and Jonas Bylander.

Received: 31 October 2013 Accepted: 6 January 2014 Published: 7 March 2014

\section{References}

1. Caves CM: Quantum limits on noise in linear amplifiers. Phys Rev D 1982, 26(8):1817-1839.

2. Feldman MJ, Parrish PT, Chiao RY: Parametric amplification by unbiased Josephson junctions. J App/ Phys 1975, 46(9):4031-4042

3. Taur Y, Richards PL: Parametric amplification and oscillation at $36 \mathrm{GHz}$ using a point-contact Josephson junction. J App/ Phys 1977, 48(3):1321-1326.

4. Feldman MJ: The thermally saturated SUPARAMP. J Appl Phys 1977, 48(3):1301-1310.

5. Wahlsten S, Rudner S, Claeson T: Parametric amplification in arrays of Josephson junctions. Appl Phys Lett 1978, 30:298-300.

6. Wahlsten S, Rudner S, Claeson T: Arrays of Josephson tunnel junctions as parametric amplifiers. J App/ Phys 1978, 49(7):4248-4263 
7. Yurke B, Kaminsky P, Miller R, Whittaker E, Smith A, Silver A, Simon R: Observation of 4.2-K equilibrium-noise squeezing via a Josephson-parametric amplifier. Phys Rev Lett 1988, 60(9):764.

8. Yurke B, Corruccini LR, Kaminsky PG, Rupp LW, Smith AD, Silver AH, Simon RW, Whittaker EA: Observation of parametric amplification and deamplification in a Josephson parametric amplifier. Phys Rev A 1989, 39(5):2519-2533.

9. Castellanos-Beltran M, Lehnert KW: Widely tunable parametric amplifier based on a superconducting quantum interference device array resonator. Appl Phys Lett 2007, 91:083509.

10. Eichler C, Bozyigit D, Lang C, Baur M, Steffen L, Fink JM, Filipp S, Wallraff A: Observation of two-mode squeezing in the microwave frequency domain. Phys Rev Lett 2011, 107(11):113601.

11. Hatridge M, Vijay R, Slichter DH, Clarke J, Siddiqi I: Dispersive magnetometry with a quantum-limited squid parametric amplifier. Phys Rev B 2011, 83(13):134501.

12. Yamamoto T, Inomata K, Watanabe M, Matsuba K, Miyazaki T, Oliver WD, Nakamura Y, Tsai JS: Flux-driven Josephson parametric amplifier. Appl Phys Lett 2008, 93(4):042510.

13. Wilson CM, Duty T, Delsing P: Parametric oscillators based on superconducting circuits. In: Fluctuating Nonlinear Oscillators. Edited by Dykman M. Oxford: Oxford University Press; 2012.

14. Sundqvist KM, Kintas S, Simoen M, Krantz P, Sandberg M, Wilson CM, Delsing P: The pumpistor: a linearized model of a flux-pumped superconducting quantum interference device for use as a negative-resistance parametric amplifier. Appl Phys Lett 2013, 103(10):102603.

15. Tholen EA, Ergul A, Doherty EM, Weber FM, Gregis F, Haviland DB: Nonlinearities and parametric amplification in superconducting coplanar waveguide resonators. Appl Phys Lett 2007, 90:253509.

16. Ho Eom B, Day PK, LeDuc HG, Zmuidzinas J: A wideband, low-noise superconducting amplifier with high dynamic range. Nat Phys 2012, 8(8):623-627.

17. Mallet F, Ong FR, Palacios-Laloy A, Nguyen F, Bertet P, Vion D, Esteve D: Single-shot qubit readout in circuit quantum electrodynamics. Nat Phys 2009, 5(11):791-795

18. Vijay R, Macklin C, Slichter DH, Weber SJ, Murch KW, Naik R, Korotkov AN, Siddiqi I: Stabilizing rabi oscillations in a superconducting qubit using quantum feedback. Nature 2012,490:77-80.

19. Flurin E, Roch N, Mallet F, Devoret $\mathrm{MH}$, Huard B: Generating entangled microwave radiation over two transmission lines. Phys Rev Lett 2012, 109(18):183901.

20. Steffen L, Salathe Y, Oppliger M, Kurpiers P, Baur M, Lang C, Eichler C, Puebla-Hellmann G, Fedorov A, Wallraff A: Deterministic quantum teleportation with feed-forward in a solid state system. Nature 2013, 500:319-322.

21. Abdo B, Schackert F, Hatridge M, Rigetti C, Devoret MH: Josephson amplifier for qubit readout. App/ Phys Lett 2011, 99(16):162506

22. Blackwell LA, Kotzebue KL: Semiconductor-Diode Parametric Amplifiers. Englewood Cliffs: Prentice Hall; 1961.

23. Decroly JC: Parametric Amplifiers. New York: Wiley; 1973.

24. Howson DP, Smith RB: Parametric Amplifiers. London: McGraw-Hill; 1970.

25. Josephson BD: Possible new effects in superconductive tunnelling. Phys Lett 1962, 1:251-253

26. Zagoskin AM: Quantum Engineering: Theory and Design of Quantum Coherent Structures. Cambridge: Cambridge University Press; 2011.

27. Van Duzer T, Turner CW: Principles of Superconductive Devices and Circuits. 2nd edition. Upper Saddle River: Prentice Hall; 1999.

28. Tinkham M: Introduction to Superconductivity. 2nd edition. New York: McGraw-Hill; 1996.

29. Maas SA: Microwave Mixers. Dedham: Artech House; 1986. [The Artech House Microwave Library]

30. Chen W-K: Active Network and Feedback Amplifier Theory. New York: McGraw-Hill; 1980.

31. Chua LO: Introduction to Nonlinear Network Theory. New York: McGraw-Hill; 1969.

32. Chua LO, Desoer CA, Kuh ES: Linear and Non-linear Circuits. New York: McGraw-Hill; 1987. [McGraw-Hill Series in Electrical and Computer Engineering.]

33. Chua LO: Nonlinear circuit foundations for nanodevices. Proc IEEE 2003, 91(11):1830-1859.

doi:10.1140/epjqt6

Cite this article as: Sundqvist and Delsing: Negative-resistance models for parametrically flux-pumped

superconducting quantum interference devices. EPJ Quantum Technology 2014 1:6.

\section{Submit your manuscript to a SpringerOpen ${ }^{\circ}$ journal and benefit from:}

- Convenient online submission

- Rigorous peer review

- Immediate publication on acceptance

- Open access: articles freely available online

- High visibility within the field

- Retaining the copyright to your article

Submit your next manuscript at $>$ springeropen.com 